\title{
White matter changes, creatinine, age, and ankle arm index predicted incident MRI defined infarctions in older adults
}

\author{
Longstreth WT Jr, Dulberg C, Manolio TA, et al. Incidence, manifestations, and predictors of brain infarcts defined by serial \\ cranial magnetic resonance imaging in the elderly: the Cardiovascular Health Study. Stroke 2002;33:2376-82.
}

\section{QUESTION: In older adults whose baseline magnetic resonance imaging (MRI) scan showed no infarctions, what are predictors of infarctions defined by serial cranial MRI?}

\section{Design}

Cohort study with mean and median follow up of 5 years (range 3.2-7.5 y).

\section{Setting}

4 communities in North Carolina, California, Maryland, and Pennsylvania, USA.

\section{Participants}

1433 adults $\geq 65$ years of age with no infarctions shown on baseline MRI scan who were able to give informed consent and to respond to questions without the aid of a surrogate respondent.

\section{Assessment of risk factors}

Baseline data on blood pressure, ankle arm index, cognitive function, activities of daily living (ADL), instrumental ADL, and depression were collected by questionnaires, physical examination, and laboratory tests.

\section{Main outcome measure}

Incident infarction defined by follow up serial cranial MRI scan, identified by neuroradiologists blinded to participants' clinical information.

\section{Main results}

On the follow up MRI scan, 254 participants (18\%) had $\geq 1$ MRI defined infarctions; of these participants, 29 (11\%) had a transient ischaemic attack or stroke between baseline and follow up scans. Of these infarctions, 192 $(76 \%)$ were single, $203(80 \%)$ were subcortical, and 221 $(87 \%)$ were $<20 \mathrm{~mm}$ in size. In a multivariate model where 9 baseline variables (with significant associations

Baseline variables associated with incident magnetic resonance imaging (MRI) defined brain infarction in older adults without infarctions shown on baseline MRI scan*

\begin{tabular}{|c|c|}
\hline Baseline variables & $\begin{array}{l}\text { Relative risk }(95 \% \text { Cl) } \\
\text { adjusted for } 9 \text { baseline } \\
\text { variables }\end{array}$ \\
\hline White matter grade & $1.57(1.40$ to 1.75$)$ \\
\hline \multirow[t]{2}{*}{ Creatinine } & 2.09 (1.21 to 3.60$)$ \\
\hline & $\begin{array}{l}\text { Relative risk }(\mathrm{Cl}) \text { adjusted } \\
\text { for baseline variables, white } \\
\text { matter grade withheld }\end{array}$ \\
\hline Creatinine & 1.77 (1.03 to 3.04$)$ \\
\hline Age & 1.04 (1.01 to 1.07$)$ \\
\hline Ankle arm index & $0.36(0.14$ to 0.88$)$ \\
\hline
\end{tabular}

*Baseline variables $=$ age, exercise, instrumental activities of daily living, systolic blood pressure, ankle-arm index, mean creatinine level, creatinine $>110.6$ $\mu \mathrm{mol} / \mathrm{l}$, white matter grade, and sulcal grade. to incident infarction) competed in stepwise fashion, severity of white matter change ("white matter grade") and serum creatinine level were predictors of incident infarction (table). In a second multivariate model where the previous variables were entered and white matter grade withheld, serum creatinine, age, and ankle arm index were predictors of incident infarction (table).

\section{Conclusion}

In older adults whose baseline magnetic resonance imaging (MRI) scan showed no infarctions, predictors of incident MRI defined infarctions were white matter grade, creatinine, age, and ankle arm index.
Source of funding: National Heart, Lung and Blood Institute.

For correspondence: Dr W T Longstreth Jr, Harborview Medical Center, Seattle, WA, USA.

wl@u.washington.edu

Abstract and commentary also appear in ACP Journal Club

\section{COMMENTARY}

Stroke or symptoms of transient brain ischaemia have long been viewed as the hallmark expression of cerebrovascular disease. Previous studies conclusively showed that risk factors for stroke are also the most common risk factors for cardiovascular and peripheral vascular disease, ${ }^{1-2}$ suggesting that these disorders share a common mechanism of vascular injury. Although much attention has focused on silent injury by cerebrovascular disease risk factors to such organ systems as the heart and the kidney, much less attention has been paid to the possible spectrum of brain injury under similar conditions. Fortunately, the advent of neuroimaging, particularly the use of population based imaging, has heralded an era of rapid growth in studies that examine the relation between cerebrovascular disease risk factors and brain infarction, even in the absence of clinical symptoms.

Although previous studies showed that white matter hyperintensities (WMHs) predict future stroke and mortality, ${ }^{3-4}$ the study by Longstreth et al is the first population based study to confirm this observation. Furthermore, the authors extend current knowledge by evaluating the combined effects of clinical as well as MRI measures. The result is a clearer picture of the many vascular risk factors that increase risk of brain infarctions by virtue of vascular injury. Using stepwise multiple regression analyses, the authors show that the extent of WMHs combined with serum creatinine levels best predict incident infarction over a mean follow up of 5 years. Removing white matter grade from the model resulted in the inclusion of age and a measure of peripheral vascular disease (ankle arm index) as additional predictors.

WMHs most likely act as surrogate markers for cerebrovascular disease. This conclusion is not new, as many associations between WMHs and various measures of atherosclerosis have been previously reported. However, it should be noted that these data suggest that the presence of WMHs on MRI scan alone may be sufficient evidence to consider stroke prophylaxis. Of course, the most prudent means to accomplish true stroke prophylaxis is early and aggressive treatment of major risk factors common to both WMH and clinical stroke, such as hypertension and diabetes.

Charles DeCarli, MD University of California, Davis Sacramento, California, USA

1 Papademetriou V, Narayan P, Rubins H, et al. Influence of risk factors on peripheral and cerebrovascular disease in men with coronary artery disease, low high-density lipoprotein cholesterol levels, and desirable low-density lipoprotein cholesterol levels. HIT Investigators. Department of Veterans Affairs HDL Intervention Trial. Am Heart J 1998;136:734-40. Weber MA. Role of hypertension in coronary artery disease. Am J Nephrol 1996;16:210-6. Miyao S, Takano A, Teramoto J, et al. Leukoaraiosis in relation to prognosis for patients with lacunar infarction. Stroke 1992;23:1434-8.

4 Streifler JY, Eliasziw M, Benavente OR, et al. Prognostic importance of leukoaraiosis in patients with symptomatic internal carotid artery stenosis. Stroke 2002;33:1651-5. 